\title{
Prevalência da doença de Darier no estado do Ceará
}

\section{Darier's disease prevalence in Brazilian Ceará state}

Levi Coelho Maia Barros ${ }^{1}$. Matheus Eugênio de Sousa Lima ${ }^{1}$. Amanda Sousa de Lima ${ }^{1}$. Daniel Gomes de Moraes Nobre'. Maria Araci Pontes Aires².

1 Discente do curso de Medicina, Universidade Estadual do Ceará (UECE), Fortaleza, Ceará, Brasil. 2 Doutora em Farmacologia Clínica, Médica Dermatologista, Diretora técnica do Centro de Referência em Dermatologia Dona Libânia, Fortaleza, Ceará, Brasil.

\section{RESUMO}

Objetivo: averiguar a situação epidemiológica da doença de Darier no estado do Ceará no ano de 2015. Metodologia: trata-se de um estudo retrospectivo quantitativo, no qual foram analisados todos os registros das consultas médicas realizadas no Centro de Referência em Dermatologia Dona Libânia (CDERM), Fortaleza, Ceará, em 2015, através das variáveis: nome, sexo, idade, data da consulta, número do prontuário, diagnóstico presuntivo ou confirmado e a frequência do paciente no CDERM. Resultados: dos 19.576 pacientes analisados, 33 pacientes foram diagnosticados com doença de Darier, representando uma incidência de $0,1685 \%$ no serviço, enquanto que na população cearense uma incidência de 1:270.000, aproximadamente. Dentre estes pacientes, 18 eram do sexo feminino e 15 do sexo masculino. A idade média dos pacientes foi de 40,66 anos. Conclusão: a menor prevalência da doença de Darier no estado do Ceará, em discordância com a literatura, no ano de 2015, poderia ser justificada por um subdiagnóstico da doença. O atraso na idade de diagnóstico corrobora para esta hipótese. Entretanto, estudos multicêntricos e epidemiológicos maiores, tanto no Ceará como no Brasil, serão necessários para uma adequada avaliação da prevalência desta doença.

Palavras-chave: Doença de Darier. Epidemiologia. Dermatologia.

\section{ABSTRACT}

Objective: Determine the epidemiological situation of Darier disease in the state of Ceará, Brazil, in the year of 2015. Methods: It's a retrospective quantitative study in which all the records of the medical consultations performed at the Centro de Referência em Dermatologia Dona Libânia (CDERM), at Fortaleza, Ceará, Brazil, in 2015, were analyzed, using the following variables: name, gender, age, date of the consultation, medical record number, presumptive or confirmed diagnosis and the patient's frequency in CDERM. Results: Of the 19,576 patients analyzed, 33 patients were diagnosed with Darier disease, representing an incidence of $0.1685 \%$ in the service, while in the population of Ceará, the prevalence is approximately 1:270,000. Among these patients, 18 were female and 15 were male. The mean age of the patients was 40.66 years. Conclusion: The lower prevalence of Darier disease in the state of Ceará, in disagreement with the literature, in the year 2015, could be justified by an underdiagnosis of the disease. The delay in the age of diagnosis corroborates this hypothesis. However, larger multicenter and epidemiological studies, both in Ceará and Brazil, will be necessary for an adequate evaluation of the prevalence of this disease.

Keywords: Darier disease. Epidemiology. Dermatology.

Autor correspondente: Levi Coelho Maia Barros, Rua Lígia Monte, 175, Cocó, Fortaleza, Ceará. CEP: 60192-370. Telefone: +55 85 99684-2168. E-mail: levicmaiabarros@gmail.com

Conflito de interesses: Não há qualquer conflito de interesses por parte de qualquer um dos autores.

Recebido em: 31 Mai 2018; Revisado em: 20 Jul 2018; Aceito em: 21 Jul 2018. 


\section{INTRODUÇÃO}

A doença de Darier (DD) foi descrita pela primeira vez em 1889. Os responsáveis por tal feito foram o dermatologista francês Jean Darier, do Hospital Saint-Louis, em Paris, e o norte-americano, professor de dermatologia da Universidade de Harvard, James C. White, os quais relataram a afecção de modo independente. Este último, no entanto, foi quem atribuiu o caráter hereditário da patologia, ao observar lesões semelhantes na filha de sua primeira paciente. ${ }^{1,2}$

Nessa perspectiva, a DD é também denominada doença de Darier-White, queratose folicular ou ainda disqueratose folicular, constituindo uma genodermatose autossômica dominante com penetrância quase completa $(95 \%))^{1-3}$ É resultado da mutação do gene ATP2A2 do cromossomo $12 \mathrm{q}$ 23-24, o que provoca um funcionamento anormal da enzima ATPase cálcio-dependente do retículo sarco/endoplasmático (SERCA-2). ${ }^{4}$ Tal fato, por sua vez, leva à alteração na sinalização de cálcio intracelular com perda da adesão suprabasal dos queratinócitos e à ativação das vias apoptóticas celulares, culminando nos achados histopatológicos de acantólise e disceratose, respectivamente. $\cdot^{3-7}$ As manifestações clínicas da doença de Darier-White ocorrem na forma de pápulas foliculares e, em alguns casos, interfoliculares. São de tonalidade amarelada-acinzentada, com tendência a coalescerem-se, resultando em placas grossas e untuosas. Os locais do corpo acometidos, predominantemente, são as regiões seborreicas, como o couro cabeludo, face, região esternal, áreas retroauriculares e temporais, tronco e dobras, possuindo frequentemente odor desagradável. Ademais, as palmas, as solas, as unhas e as mucosas podem também ser atingidas..$^{3,7,8}$

Paralelamente a isso, a disceratose folicular apresenta, ainda, diferentes classificações clínicas descritas, tais como as variações vesículobolhosa, comedônica, acral hemorrágica, localizada linear, leucodérmica macular e a cornificada. Nesse ponto de vista, a DD localizada pode ser dividida em dois subtipos: Tipos 1 e 2. De modo que no primeiro (tipo 1), as lesões seguem as linhas de Blaschko unilateralmente, sem outras manifestações da doença clássica. No segundo (tipo 2), por sua vez, tais lesões constituem regiões focais de gravidade maior, sobreposta à DD disseminada (clássica). A prevalência do tipo linear não é conhecida, estima-se, entretanto, que ela corresponda a $10 \%$ de todos os casos de DD. ${ }^{2,9}$

Os dados na literatura acerca da epidemiologia dessa doença são escassos mundialmente, e quando a busca se refina em direção ao Brasil, tornam-se raros. Nesse aspecto, não foram encontradas, na literatura, pesquisas no estado do Ceará que abordassem a DD. Esse fato é lamentável, já que a escassez de pesquisas sobre uma determinada afecção provoca, entre outros prejuízos, a perda de importantes informações sobre as variáveis vinculadas ao perfil populacional mais atingido por uma patologia (como idade, sexo, prevalência e recorrência), dificultando, dessa maneira, um efetivo desenvolvimento da epidemiologia brasileira. ${ }^{10}$
Diante disso, expõe-se a necessidade de mais estudos nacionais e regionais sobre as características epidemiológicas da DD, a fim de serem traçados panoramas atuais sobre essa afecção. Tal fato permitirá, dentre outros fatores, sua melhor compreensão por parte de governo, sociedade e comunidade científica, colaborando, dessa forma, para a elaboração de políticas públicas de saúde cabíveis, atenuação do estigma social e definição de tratamentos que visem proporcionar uma melhor qualidade de vida aos indivíduos portadores dessa genodermatose. ${ }^{11}$

\section{MATERIAIS E MÉTODOS}

Trata-se de um estudo retrospectivo quantitativo, no qual foram analisados todos os registros das consultas médicas realizadas no Centro de Referência em Dermatologia Dona Libânia (CDERM), Fortaleza, Ceará, no período de janeiro a dezembro de 2015.

Os registros manuscritos disponibilizavam as seguintes variáveis: nome, sexo, idade, data da consulta, número do prontuário, diagnóstico presuntivo ou confirmado e a frequência do paciente no CDERM (se primeira vez ou retorno ao atendimento médico da instituição). Baseado na variável nome, foram atendidos 19.576 pacientes no período estipulado.

Foram excluídos os pacientes cuja identificação foi prejudicada pelos registros manuscritos de má qualidade, além daqueles atendidos por profissionais da saúde não médicos. Contabilizou-se o número de retornos ao CDERM.

Destes registros, foram selecionados aqueles cujo diagnóstico confirmado foi de doença de Darier, a fim de conhecer sua incidência neste serviço de referência e analisar suas variáveis a procura de relações causais.

Os dados foram colhidos durante o período de outubro de 2016 a junho de 2017. As variáveis foram digitalizadas no software Microsoft Office Excel ${ }^{\circledR}$, onde foram submetidas à análise estatística com testes paramétricos.

O projeto de pesquisa foi enviado e aprovado pelo Comitê de Ética do CDERM, de acordo com os ditames éticos de respeito ao sigilo e à autonomia do paciente. Não há conflito de interesses nesse trabalho.

\section{RESULTADOS}

Dos 19.576 pacientes analisados, 33 pacientes foram diagnosticados com DD, representando uma incidência de 0,1685\% no serviço. Dentre estes pacientes, 18 (54,55\%) eram do sexo feminino e $15(45,45 \%)$ do sexo masculino. A idade média dos pacientes foi de 40,66 anos. A idade detalhada está representada na Tabela 1 .

A recorrência ao serviço variou, como pode ser visto na Tabela 2. Foram realizadas um total de 108 consultas ao 
longo do ano com esses pacientes. Nenhum deles procurou o CDERM por outras queixas que não a DD. A grande maioria dos pacientes $(87,88 \%)$ foi reconsultado pelo menos uma vez ao longo do ano.

Tabela 1. Idade e sexo dos pacientes com DD.

\begin{tabular}{ccccc}
\hline Idade & Número absoluto & $\%$ & $\%$ Masculino & $\%$ Feminino \\
\hline $0-10$ & 0 & 0 & 0 & 0 \\
$11-20$ & 4 & 12 & 80 & 20 \\
$21-30$ & 7 & 21 & 57,14 & 42,86 \\
$31-40$ & 6 & 21 & 33,33 & 66,67 \\
$41-50$ & 6 & 18 & 33,33 & 66,67 \\
$51-60$ & 6 & 18 & 33,33 & 66,67 \\
$61-70$ & 0 & 0 & 0 & 0 \\
$>70$ & 4 & 12 & 50 & 50 \\
\hline
\end{tabular}

Tabela 2. Recorrência das consultas.

\begin{tabular}{cc}
\hline $\mathrm{N}^{\mathrm{o}}$ consultas & Pacientes \\
\hline 1 & 4 \\
2 & 8 \\
3 & 7 \\
4 & 4 \\
5 & 9 \\
6 & 1 \\
\hline
\end{tabular}

\section{DISCUSSÃO}

A DD é uma doença rara, possuindo uma prevalência estimada de 1 em 55.000 à 100.000 indivíduos. ${ }^{6}$ De acordo com outros autores, tal prevalência revela um intervalo maior de 1 em 30.000 à 100.000 pessoas, como apresentado na Escandinávia e na Inglaterra. ${ }^{4,8,12}$ Em países como a Argentina, por outro lado, a frequência dos casos da doença é desconhecida. ${ }^{2}$ Baseado nos dados do IBGE a respeito da população cearense no ano de $2015,{ }^{13}$ a DD teria prevalência de 1:270.000 indivíduos, aproximadamente. Este número, discordante na literatura, poderia indicar um subdiagnóstico da doença, um encaminhamento de doentes para outros serviços especializados em dermatologia no Ceará ou mesmo uma menor prevalência da doença no estado em relação à prevalência global.

No nosso estudo, a incidência da doença no número de atendimentos foi de 1:600, aproximadamente, evidenciando o caráter especializado do CDERM, que por concentrar pacientes com enfermidades dermatológicas mais raras acaba por aumentar sua real prevalência na sociedade. Em relação ao gênero, nosso estudo é consoante com a literatura de que a doença de Darier afeta igualmente homens e mulheres. ${ }^{1,6,8}$ Em homens, no entanto, as manifestações clínicas mais severas são mais comuns que em mulheres. ${ }^{14}$

No que tange à faixa etária atingida, em cerca de $70 \%$ dos casos o aparecimento dos primeiros sinais da patologia ocorre entre 6 e 20 anos de idade, ${ }^{1,6}$ muito embora alguns casos possam ocorrer no final da vida adulta. ${ }^{15}$ Tal fato, entretanto, não está em concordância com os resultados encontrados no presente estudo, no qual, dos 33 casos, apenas 4 (12\%) foram diagnosticados até a segunda década de vida. Essa discrepância pode dever-se à ausência da informação da data inicial do diagnóstico nos documentos analisados. Ainda assim, tal dado nos permite interrogar se a DD no estado do Ceará ocorre de forma mais tardia que o relatado pela literatura internacional ou se há um importante atraso diagnóstico dessa enfermidade no contexto cearense.

No Brasil, há alguns relatos de caso sobre a DD. Em um desses estudos, realizado em 1941, buscou-se identificar os primeiros casos da DD no estado de Pernambuco. Na época, poucos casos haviam sido descritos no mundo. ${ }^{16}$ Hoje, mais de 75 anos depois, ainda não há informações epidemiológicas suficientes e de fácil acesso para a comunidade científica, ressaltando-se a necessidade de melhor conhecer a DD e suas características, a fim de incluí-la em diagnósticos diferenciais e, assim, permitir o correto diagnóstico e manejo de indivíduos portadores dessa enfermidade.

Uma rara pesquisa sobre essa doença foi realizada por meio de um estudo prospectivo na cidade de São Paulo, que avaliou 313 consultas dermatológicas em um hospital universitário. Nestas, os diagnósticos mais prevalentes foram de doenças infecciosas e parasitárias $(26,8 \%)$, enquanto da DD foram observados 2 casos $(0,6 \%) .{ }^{17}$ Em nosso estudo, foram observados 33 casos $(0,1685 \%)$, incidência inferior à observada na pesquisa realizada na cidade de São Paulo, entretanto, para comparação mais efetiva, é de suma importância ampliar o número de casos analisados em estudos realizados em outros estados.

Outro estudo, realizado no Rio de Janeiro, destaca a dificuldade de diagnosticar a DD, principalmente em sua forma localizada que, apesar de sua baixa gravidade, pode gerar desconforto ao paciente. Destaca-se que se deve pensar em DD quando da presença de lesões que seguem as linhas de Blaschko e da importância do exame histopatológico para firmar o diagnóstico. ${ }^{6}$ São relatados como fatores que auxiliam o diagnóstico clínico a queixa de prurido, com exacerbações atribuídas ao calor, transpiração, luz solar, tratamento de esteroides, estresse e menstruação. ${ }^{15}$ Nos documentos analisados em nosso estudo, não há referências à exames histopatológicos.

No estado do Ceará, não foram localizados estudos publicados que discorressem sobre a epidemiologia da DD. Assim, concluímos reiterando a necessidade de mais estudos com esse enfoque, de forma a ampliar o conhecimento médico e possibilitar avanços, tanto na área médica, como na melhoria da qualidade de vida desses pacientes. 


\section{REFERÊNCIAS}

1. Hohl, D. Doença de Darier e doença de Hailey-Hailey. In: Bolognia JL, Jorizzo JL, Schaffer JV. Dermatologia. 3. ed. Rio de Janeiro: Elsevier; 2015. p.887-98.

2. Lequio M, Garrido MA, Giavino GV, Vigovich F, Chouela EN. Enfermedad de Darier diseminada. Dermatología Argentina. 2011;17(6):470-3.

3. Linder D, Marinello E, Donisi PM, Salmaso R, Zattra E, Zampetti A. Inframammary dermatitis: a case of localized late-onset Darier's disease. Case Rep Dermatol. 2016;8(2):189-92.

4. Mestre T, Pacheco FA, Lázaro A, Cardoso J. Doença de Darier comedónica. Revista da Sociedade Portuguesa de Dermatologia e Venereologia. 2015;73(3):389-92.

5. Quaresma MV, Abulafia LA. Genodermatoses acantolíticas com disceratose e doenças relacionadas. In: Azulay RD, Azulay DR, Abulafia LA. Dermatologia. 6. ed. Rio de Janeiro: Guanabara Koogan; 2013. p.854-6.

6. Medeiros PM, Alves NR, Trujillo JM, Silva CC, Faria PC, Silva RS. Segmental Darier's disease: a presentation of difficult diagnosis. An Bras Dermatol. 2015;90(3 Supl 1):62-5.

7. Saul LM, Neglia V, Kien MC, Abeldaño A. Enfermedad de Darier: comunicación de 4 casos. Arch Argent Dermatol. 2015;65(1):22-7.

8. Castagna J, Clerc C, Dupond AS. Exacerbation of Darier disease by peg-interferon beta-1a in a patient suffering from multiple sclerosis. Int J Dermatol. 2018;57(1):116-7.

9. Miguel D, Schliemann S, Brautzsch I, Elsner P. Occupational Darier's disease. J Dtsch Dermatol Ges. 2018;16(1):64-5.
10. Teixeira MG. IV Plano diretor para o desenvolvimento da epidemiologia no Brasil. Rev Bras Epidemiol. 2005;8(3):231-3.

11. Brasil. Ministério da Saúde. Diretrizes para Atenção Integral às Pessoas com Doenças Raras no âmbito do Sistema Único de Saúde (SUS) [Internet]. Brasília (DF): Ministério da Saúde; 2014 [acesso em: 04 maio 2018]. Disponível em: http://bvsms.saude.gov.br/bvs/ publicacoes/diretrizes_atencao_integral_pessoa_doencas_raras SUS.pdf

12. Leong IU, Stuckey A, Ahanian T, Cederlöf M, Wikstrom JD. Novel mutations in Darier disease and association to self-reported disease severity. PloS one. 2017;12(10):e0186356.

13. Instituto Brasileiro de Geografia e Estatística. Estimativas populacionais para os municípios e para as Unidades da Federação brasileiros em 01.07.2015 [Internet]. Rio de Janeiro: IBGE; 2015 [acesso em 27 mar 2018]. Disponível em: https://ww2.ibge.gov.br/ home/estatistica/populacao/estimativa2015/estimativa_dou.shtm

14. Ferizi M, Begolli-Gerqari A, Luzar B, Kurshumliu F, Ferizi M. A rare clinical presentation of Darier's disease. Case Rep Dermatol Med. 2013;2013:419797

15. Cooper SM, Burge SM. Darier's disease. Am J Clin Dermatol. 2003;4(2):97-105.

16. Lobo J, Rocha G. Doença de Darier. An Bras Dermatol. 1942;17(2):pag.11-14

17. Mancusi S, Festa C Neto. Inpatient dermatological consultations in a university hospital. Clinics (São Paulo). 2010;65(9):851-5.

\section{Como citar:}

Barros LC, Lima ME, Lima AS, Nobre DG, Aires MA. Prevalência da doença de Darier no estado do Ceará. Rev Med UFC. 2019 jan-mar;59(1):30-33. 